\title{
Relación entre los estilos del humor y la satisfacción con la vida en estudiantes de Secundaria
}

\author{
Carlos Salavera y Pablo Usán \\ Universidad de Zaragoza (España)
}

\begin{abstract}
Se evaluó a 1304 estudiantes de Secundaria (12-17 años) y se analizaron los estilos del humor y su satisfacción con la vida. Los resultados muestran unos adolescentes moderadamente satisfechos con su vida y que utilizan los estilos de humor en este orden: afiliativo, automejora, agresivo y contraproducente. La satisfacción con la vida correlacionó con los estilos positivos del humor (afiliativo y automejora) e inversamente con el uso del humor agresivo. Apenas un $25.85 \%$ de los estudiantes de la muestra, hacen un buen uso del humor (mayor uso de los estilos positivos y menor de los estilos negativos) y muestran buenos índices de satisfacción con la vida. Además, aunque los hombres hacen un mayor uso del humor, tanto positivo como negativo, ser mujer aumenta la satisfacción con la vida en 0.85 puntos frente a ser hombre, siendo el género la variable de mayor peso en la predicción de la puntuación en satisfacción con la vida, seguida de los humores de automejora, agresivo y afiliativo. Como conclusión, los datos de la investigación proporcionan un perfil de adolescentes moderadamente satisfechos con su vida, con un mayor uso de los estilos de humor positivos, así como evidencias de la relación entre los estilos del humor y la satisfacción con la vida.
\end{abstract}

Palabras clave: Estilos del humor, satisfacción con la vida, adolescentes.

Relationship between the use of humor and life satisfaction in Secondary School students. Secondary school students $(N=1304)$ and $12-17$ years old, were evaluated and mood styles and their satisfaction with life were analyzed. The results show a moderately satisfied with their lives teenagers and humor styles are used in this order: affiliative, self-improvement, aggressive and self-destructive. The life satisfaction correlated with positive styles of humor (affiliative and self-improvement) and inversely with the use of aggressive humor. Just a $25.85 \%$ of adolescents in the sample, make good use of humor (greater use of positive and less negative styles) and good levels of satisfaction with life. In addition, although men make more use of humor, both positive and negative, being a woman increases life satisfaction by 0.85 points from being male, being gender variable greater weight in predicting the score life satisfaction, self-improvement followed humor, aggressive humor and affiliative humor. In conclusion, stating that the research data provide a profile of moderately satisfied with his life teenagers, with greater use of the styles of positive humor, as well as evidence of the relationship between the use of humor and life satisfaction.

Keywords: Styles of humor, satisfaction with life, adolescents.

Correspondencia: Carlos Salavera. Departamento de Psicología y Sociología. Universidad de Zaragoza. C.P.: 50009. Zaragoza (España). E-mail: salavera@unizar.es 
El uso del humor ha cobrado especial importancia en los últimos años debido a su relación con la salud física y psicológica de la persona (Capps, 2006; Kuiper, 2014), encontrándose relación con la reducción del dolor, mejora de la condición física y mejores niveles de salud en general (Cann y Collette, 2014; Panish, 2002). El humor está implicado en algunas de las tareas psicológicas más complejas e importantes, siendo responsable de distintos beneficios sociales, cognitivos y del comportamiento, tanto en tareas individuales como en aspectos interpersonales (Carretero-Dios, Benítez, DelgadoRico, Ruch, y López-Benítez, 2014; Cassaretto, y Martínez, 2009; Gignac, Karatamoglou, Wee, y Palacios, 2014; Kozbetz y Nishioka, 2010; Martin, 2003; Sirigatti, Penzo, Giannetti, Casale, y Stefanile, 2016). Así, podemos hablar de diferentes estilos de humor (Martin, 2001), dos que son a priori potencialmente beneficiosos para la persona que los posee: afiliativo (uso benigno del humor para facilitar las relaciones interpersonales) y automejora (empleo de una visión humorística pero realista de la vida); y dos que son considerados potencialmente perjudiciales: agresivo (utilizado para sobresalir a expensas de otros y contraproducente (uso de un humor cínico divirtiendo a los demás a expensas de la propia persona).

Algunos autores han sugerido que el humor juega un papel importante en las relaciones interpersonales, que puede estar relacionadas con la satisfacción con la vida (Aillaud y Piolat, 2013; Kuiper, Grimshaw, Leite, y Kirsh, 2004; Maiolin y Kuiper, 2014).

Esta idea parte de la investigación sobre el constructo de bienestar subjetivo (Diener, 1984; Diener y Larsen, 1993; Heintz, 2017; Smedema, Catalano, y Ebener, 2010). En este constructo se identifican dos componentes claramente diferenciados; un componente emocional, con dos subcomponentes: afectos positivos y afectos negativos (Lucas, Diener, y Suh, 1996; Sandin, Chorot, Lostao, Joiner, Santed, y Valiente, 1999), y un componente cognitivo, denominado satisfacción con la vida (Andrews y Withey, 1976).

En cuanto a este componente cognitivo, la satisfacción con la vida se define como una evaluación global que la persona hace sobre su vida. Para ello, la persona evalúa aspectos tangibles de su vida, sopesa lo bueno y lo malo, comparándolo con estándares elegidos por ella (Shin y Johnson, 1978), y realiza un juicio sobre la satisfacción con su vida (Pavot, Diener, Colvin, y Sandvik, 1991). Los resultados de esta satisfacción dependen de las comparaciones que el sujeto hace entre las circunstancias de su vida y el estándar que considera apropiado. Este último matiz es importante ya que no se trata de un estándar impuesto externamente sino que es un criterio auto impuesto (Atienza, Pons, Balaguer, y García-Merita, 2000). Según Diener (1984) los trabajos sobre la satisfacción con la vida se deben centrar en los juicios subjetivos que hace la persona sobre su propia vida. Por tanto, es necesario preguntarle a la persona por una evaluación global sobre su vida, así lo consideraron Diener, Emmons, Larsen, y Griffin 
(1985), quienes consideraron necesario construir una escala que evaluara la satisfacción con la vida como un proceso de juicio cognitivo.

El objetivo de esta investigación ha estado centrado en el análisis de los estilos del humor y la satisfacción con la vida, además de la relación de esta última con los diferentes estilos de humor (afiliativo, de automejora, agresivo y autoagresivo).

El estudio presenta dos hipótesis de partida: 1) los estilos del humor y la satisfacción con la vida de los adolescentes, se encuentran relacionados; y 2) los estilos del humor utilizados por el sujeto, predicen la satisfacción con la vida de la persona.

\section{MÉTODO}

\section{Participantes}

El muestreo realizado fue de tipo estratificado siendo la unidad primaria la titularidad de los centros y la etapa de Secundaria el nivel educativo $\left(1^{\circ}, 2^{\circ}, 3^{\circ}\right.$ y $4^{\circ}$ ESO). La muestra estuvo formada por 1304 adolescentes: 647 hombres (49.61\%) у 657 mujeres (50.38\%), de distintos Institutos de Educación Secundaria. La edad de los participantes fue de 12 a 17 años, con una media de 14.89 años $(s=1.598)$. Todos ellos participaron en el estudio de manera voluntaria, firmaron el consentimiento informado y se observaron las consideraciones éticas de la Declaración de Helsinki y cumplió los criterios éticos de investigación con seres humanos (consentimiento informado de padres y madres, profesores y alumnos y derecho de información, protección de datos personales y garantías de confidencialidad, no discriminación, gratuidad y posibilidad de abandonar el estudio en cualquiera de sus fases). Realizado el cálculo de la representatividad de la muestra, con un nivel de confianza del $99 \%$ y un error muestral del $5 \%$, se obtuvo que la muestra final encuestada resultó representativa de la provincia de Zaragoza. El estudio se diseñó como un estudio retrospectivo ex-post facto (Montero y León, 2007).

Tabla 1. Distribución de la muestra por edades

\begin{tabular}{cccccc}
\hline Edad & Frecuencia & Porcentaje & Edad & Frecuencia & Porcentaje \\
\hline 12 & 203 & $15.57 \%$ & 15 & 327 & $25,07 \%$ \\
\hline 13 & 324 & $24.85 \%$ & 16 & 94 & $7.43 \%$ \\
\hline 14 & 329 & $25.23 \%$ & 17 & 24 & $1.84 \%$ \\
\hline
\end{tabular}

\section{Instrumentos}

Cuestionario de estilos de humor (HSQ; Martin, Publio-Doris, Larsen, Gray, y Weir, 2003). Este cuestionario de estilos de humor, tiene formato de autoinforme con 32 ítems, de escala Likert de 7 puntos, que señala cuatro estilos de humor: dos positivos (afiliación, uso benigno del humor para facilitar las relaciones interpersonales; automejora: empleo de una visión humorística pero realista de la vida) y otros dos 
negativos (agresivo: utilizado para sobresalir a expensas de otros; y contraproducente: uso de un humor cínico divirtiendo a los demás a expensas de la propia persona). Posee una fiabilidad alta $(\alpha=0.82)$ en el presente estudio. Los coeficientes de fiabilidad (alfa de Cronbach) en las diferentes escalas fueron: Afiliativo .82; Automejora .85; Agresivo .78; y Contraproducente .82.

Escala de Satisfacción con la Vida (SWLS; Diener, Emmons, Larsen, y Griffin, 1985). Consiste en un conjunto de 5 afirmaciones frente a las cuales las personas deben indicar su nivel de acuerdo o desacuerdo en una escala tipo Likert de 7 puntos (desde $1=$ fuertemente en desacuerdo a 7 =fuertemente de acuerdo). Las puntuaciones pueden oscilar entre 5 y 35 puntos, indicando una mayor satisfacción vital las puntuaciones más altas. Esta escala posee una alta consistencia interna, con un coeficiente alfa de Cronbach de 0.87 en esta investigación.

\section{Diseño y procedimiento}

Los participantes tenían treinta minutos para completar los cuestionarios que se acaban de describir y el consentimiento informado. En todos los casos se les recordaba que la información recogida era anónima y confidencial.

Los datos recogidos en el presente estudio se obtuvieron entre los meses de marzo a mayo de 2016.

Para el análisis estadístico de los datos se ha empleado el programa estadístico SPSS 22.0. Se realizó un análisis descriptivo para cada una de las variables. En todos los casos se trabajó con el menor nivel de significación posible y se han considerado significativas aquellas diferencias con un valor de $p<0.05$. Los contrastes se plantearon de forma bilateral. Se estableció un conglomerado de medias para obtener clúster que nos permitiesen clasificar a los participantes en distintos grupos en función de la utilización del humor y satisfacción con la vida. Finalmente, el establecimiento de ecuaciones predictoras de la satisfacción con la vida en función del uso del humor, se realizó mediante la técnica de regresión logística, siguiendo el procedimiento de regresión por pasos hacia delante basado en el estadístico de Wald. Finalmente, con el programa estadístico AMOS v24, se planteó un modelo de ecuaciones estructurales que permitiese validar y cuantificar relaciones causales entre autoeficacia, habilidades sociales e inteligencia emocional a partir de los datos observados en la investigación.

\section{RESULTADOS}

En primer lugar, se realizaron análisis descriptivos de la muestra. Esto indican que los alumnos de la muestra se consideran a sí mismos moderadamente satisfechos con la vida $(x=4,96 ; s=1,01)$, en una escala de 1 a 7 . Del mismo modo, el uso del humor que utilizan lo indican las puntuaciones de uso de los distintos usos de humor: humor 
afiliativo $(\chi=31.81 ; s=4.45)$, humor de automejora $(\chi=26.38 ; s=5.10)$, humor agresivo $(\chi=18.98 ; s=3.93)$ y humor contraproducente $(\chi=16.99 ; s=4.56)$. Lo que nos indica que los adolescentes de la muestra hacen un mayor uso, en términos generales, de los estilos de humor positivos.

También se analizaron las diferencias en cuanto a género en uso del humor y satisfacción con la vida (Tabla 2). Se encontraron diferencias significativas en este sentido en todos los tipos de humor, así como en satisfacción con la vida.

En los distintos factores de humor, las puntuaciones indican un mayor uso del humor en sus cuatro factores por parte de los hombres, tanto en los estilos de humor positivos (afiliativo y automejora), como en los negativos (agresivo y contraproducente). Las mujeres muestran una mayor puntuación en la satisfacción con la vida. Los resultados, muestran como tanto los estilos del humor como la satisfacción con la vida obtienen diferencias significativas respecto al género.

Tabla 2. Resultados por género en las escalas HSQ y SWLS

\begin{tabular}{llcccccc}
\hline \multirow{2}{*}{} & & \multicolumn{3}{c}{ Hombres } & \multicolumn{2}{c}{ Mujeres } & \multirow{2}{*}{ Sig. } \\
\cline { 3 - 6 } & & $\chi$ & $s$ & $\chi$ & $s$ & & \\
\hline \multirow{4}{*}{ HSQ } & Humor afiliativo & 33.62 & 3.92 & 31.44 & 4.47 & 182.154 & .000 \\
& Humor automejora & 28.29 & 5.00 & 25.99 & 5.03 & 132.077 & .000 \\
& Humor agresivo & 21.01 & 3.58 & 18.56 & 3.87 & 123.234 & .000 \\
& Humor autodestructivo & 20.08 & 4.74 & 16.36 & 4.25 & 95.171 & .000 \\
\hline SWLS & & 4.25 & 1.08 & 5.11 & .94 & 7.410 & .007 \\
\hline
\end{tabular}

La tabla 3 presenta las correlaciones parciales entre la escala del Humor (HSQ) y satisfacción con la vida. La satisfacción con la vida correlacionó con los estilos positivos del humor: afiliativo $\left(p=.180^{*}\right)$ y automejora $\left(p=.215^{*}\right)$ y de manera inversa con el humor agresivo $(p=-.199 *)$, lo que nos indicaría que el uso del humor positivo y un bajo uso del humor agresivo, influye en la satisfacción de la persona con la vida.

Tabla 3. Correlaciones de las dimensiones humor y satisfacción con la vida.
\begin{tabular}{lccccc}
\hline Variable & 1 & 2 & 3 & 4 \\
\hline 1. Humor afiliativo & 1 & & & \\
2. Humor automejora & $.340^{* *}$ & 1 & & \\
3. Humor agresivo & .152 & $.180^{*}$ & 1 & \\
4. Humor autodestructivo & .099 & $.202^{*}$ & $.341^{* *}$ & 1 \\
5. Satisfacción con la vida & $.180^{*}$ & $.215^{*}$ & $-.199^{*}$ & -.12 \\
\hline$* p<.05 ; * * p<.01$
\end{tabular}

Además, se realizó un análisis de clúster (Tabla 4) para intentar clasificar a los participantes en la investigación en función de las variables uso del humor y satisfacción con la vida. De esta manera, se obtuvieron tres grupos: 1) formado por 302 sujetos $(23.16 \%)$, con un mayor uso del humor en todos sus apartados: afiliativo, automejora, agresivo y contraproducente y una satisfacción con la vida inferior a la media de la muestra; 2) con 665 sujetos $(50.99 \%)$ que mostraban niveles más bajos en el uso del 
humor, tanto en los estilos positivos como negativos y con un nivel más bajo en el apartado de satisfacción con la vida; y 3) con 337 participantes (25.85\%) en los que se encontraron niveles más altos de uso de los humores positivos (afiliativo y de automejora), menor uso del humor contraproducente y un índice mayor de satisfacción con la vida.

Tabla 4. Centro de conglomerados finales de las variables estilos del humor y satisfacción con la vida

\begin{tabular}{llcccc}
\hline & & 1 & 2 & 3 & $\chi$ \\
\hline \multirow{4}{*}{ HSQ } & Afiliativo & $34.36^{* * *}$ & $29.41^{*}$ & $34.25^{* * *}$ & $31.81^{* *}$ \\
& Automejora & $29.36^{* * *}$ & $22.80^{*}$ & $30.76^{* * *}$ & $26.38^{* *}$ \\
& Agresivo & $21.15^{* * *}$ & $17.92^{*}$ & $19.13^{* *}$ & $18.98^{* *}$ \\
& Autodestructivo & $22.57^{* * *}$ & $15.73^{*}$ & $14.46^{*}$ & $16.99^{* *}$ \\
\hline SWLS & $4.90^{*}$ & $4.85^{*}$ & $5.26^{* * *}$ & $5.11^{* *}$ \\
\hline $\mathrm{N}^{\circ}$ sujetos & 302 & 665 & 337 \\
Porcentaje & $(23.16 \%)$ & $(50.99 \%)$ & $(25.85 \%)$ \\
\hline *Valores por debajo de la media; $* *$ Valores próximos a la media; ***Valores por \\
encima de la media
\end{tabular}

Por último, se intentó contrastar la segunda hipótesis los estilos del humor utilizados por el sujeto, predicen la satisfacción con la vida de la persona. Para ello, se realizó una regresión múltiple seleccionando como variables predictoras las puntuaciones factoriales correspondientes a uso del humor y como variable criterio la satisfacción con la vida, para precisar el valor predictivo del uso del humor sobre ésta. La tabla 5 muestra los pasos seguidos por los modelos en la introducción de las variables explicativas que han resultado significativas para la predicción de la probabilidad de satisfacción con la vida.

Tabla 5. Análisis de regresión múltiple sobre la satisfacción con la vida

\begin{tabular}{|c|c|c|c|c|c|c|}
\hline \multirow{2}{*}{ Predictor } & \multicolumn{3}{|c|}{ Paso 1} & \multicolumn{3}{|c|}{ Paso 2} \\
\hline & $B(\mathrm{ET})$ & $t$ & $r$ parcial & $B(\mathrm{ET})$ & $t$ & $r$ parcial \\
\hline Género & $0.81(0.22)$ & $3.64 * * *$ & 0,30 & $0.85(0.23)$ & $3.77 * * *$ & 0.31 \\
\hline Edad & $-0.02(0.02)$ & -1.15 & $-0,10$ & $-0.02(0.02)$ & -1.17 & -0.10 \\
\hline 1. Humor afiliativo & & & & $0.03(0.01)$ & $2.12 *$ & 0.18 \\
\hline 2. Humor automejora & & & & $0.04(0.01)$ & $2.92 * *$ & 0.25 \\
\hline 3. Humor agresivo & & & & $-0.04(0.02)$ & $-2.39 *$ & -0.21 \\
\hline 4. Humor autodestructivo & & & & $0.00(0.01)$ & -0.17 & -0.02 \\
\hline $\mathrm{R}^{2}(\%)$ & \multicolumn{3}{|c|}{10.9} & \multicolumn{3}{|c|}{24.0} \\
\hline$\Delta \mathrm{R}^{2}(\%)$ & \multirow{2}{*}{\multicolumn{3}{|c|}{$F_{(10.1304)}=2.20 * * *$}} & \multicolumn{3}{|c|}{13.1} \\
\hline Modelo & & & & $F_{(10.1}$ & $304)=2.20 *$ & \\
\hline
\end{tabular}

Como se puede observar la variable género presentó efecto directo y significativo sobre la satisfacción (paso1). Con la inclusión en el modelo (paso 2) de las dimensiones del humor se produjo un aumento significativo de la varianza explicada del $13.1 \%$, siendo el total de la misma del $24 \%$. En este modelo el género mostró un efecto directo y significativo $(p<.001)$ con la satisfacción de forma que ser mujer aumenta la satisfacción en 0.85 puntos frente a ser hombre. Con respecto al humor, el afiliativo y el de automejora mostraron un efecto directo y significativo por lo que la puntuación en la 
satisfacción se asocia a niveles altos de dichos tipos de humor: afiliativo $(B=.03, p=.036)$ $\mathrm{y}$ automejora $(B=.04, p=.004)$. El humor agresivo mostró un efecto indirecto $\mathrm{y}$ significativo por lo que la puntuación en la satisfacción se asocia a niveles bajos de dicho humor $(B=-.04, p=.018)$. Según los coeficientes de correlación parciales, el género $(r=.31)$ y el humor de automejora $(r=.25)$ son las variables de mayor peso en la predicción de la puntuación en satisfacción, seguidas del humor agresivo $(r=-.21)$ y el humor afiliativo $(r=.18)$.

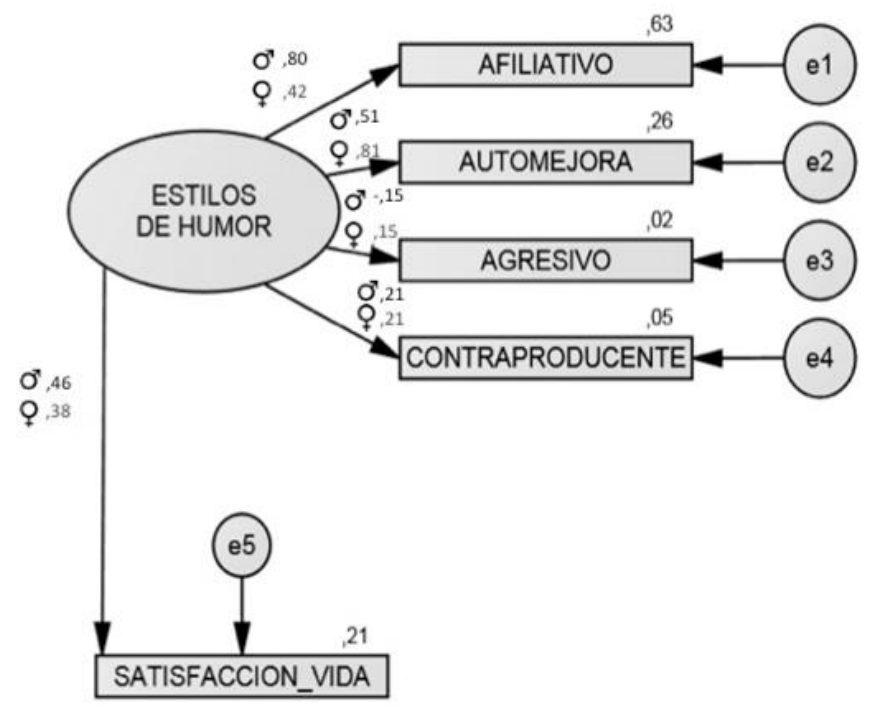

Figura 1. Análisis del modelo compuesto

En esta misma línea de análisis, la figura 1 muestra el resultado del análisis con ecuaciones estructurales mediante el método de extracción de máxima verosimilitud, confirmando la adecuación del modelo compuesto por los constructos planteados en el estudio. En este caso, al analizarlo teniendo en cuenta el género, se encontraron diferencias, siendo en el caso de los hombres, algo mayor el peso de los estilos del humor en la satisfacción con la vida, cuando los analizamos de manera global. Por factores, el humor afiliativo tiene una mayor correlación con los estilos del humor en los hombres, presentando los otros tres estilos (automejora, agresivo y contraproducente) mayor peso en esta variable en el caso de las mujeres. Por lo que se refiere al ajuste del modelo, los diversos índices de ajuste resultaron adecuados, por lo que se puede afirmar que el modelo propuesto acerca de la estructura factorial entre los tres factores es sustentable: $\quad \chi_{(10)}^{2}=2.01 \quad p<0.001 ; \quad \chi^{2} / \mathrm{gl}=2.20 ; \quad \mathrm{CFI}=0.89 ; \quad \mathrm{NFI}=0.86 ; \quad \mathrm{TLI}=0.81$; RMSEA=0.071, IC 95\%(0.065-0.084). 


\section{DISCUSIÓN Y CONCLUSIONES}

En la investigación se encontró que los adolescentes muestran una cierta satisfacción con la vida, su puntuación media fue de 4.96 sobre 7 . Hacen un mayor uso del humor afiliativo y de automejora, que son considerados estilos de humor positivos. Por el contrario, los estilos agresivo y contraproducente obtuvieron bajas puntuaciones, indicando un uso más bajo en los participantes del estudio. Apenas un 25,85\% de los adolescentes de la muestra, hacen un buen uso del humor (mayor uso de los estilos positivos y menor de los estilos negativos) que influye en buenos índices de satisfacción con la vida. El resto de los participantes $(74.15 \%)$ muestran un mayor $(23.16 \%)$ o menor uso $(50.99 \%)$ de los estilos de humor (tanto positivos como negativos) y su satisfacción con la vida es menor.

Los datos indican como los hombres hacen un uso más elevado de los distintos estilos del humor, tantos positivos como negativos, en línea con algunos estudios recientes (Dyck y Holtzman, 2013; Walther y Hilbert, 2015), aun cuanto esto supone una diferencia con otros estudios que no encontraron diferencias en cuanto al uso de las emociones en cuestión de género (Batool, Niazi, y Ghayas, 2014; Hay, 2000; Kotthoff, 2006), o que señalaban un mejor manejo de las emociones en el caso de las mujeres (Extremera, Fernández-Berrocal, y Salovey, 2006), si bien señalar como la variable género si mostró influencia con los usos del humor y con la variable satisfacción con la vida.

En cuanto al uso del humor, se encontró correspondencia, con puntuaciones similares a estudios anteriores (Capps, 2006; Goma y Valero, 2008; Yip y Martin, 2006). Estos resultados se pueden explicar, dado que el humor afiliativo (o humor social) mide el uso benigno del humor para facilitar las relaciones interpersonales (Erickson y Feldstein, 2007), lo que implicaría su relación con la gestión de las emociones, en este caso la satisfacción con la vida, en línea de los datos encontrados en otras investigaciones anteriores (Kuiper y McHale, 2009). El humor de automejora, que mide el empleo de una visión humorística pero realista de la vida y que se usa como una estrategia de afrontamiento para regular las emociones negativas (Erickson y Feldstein, 2007; Saroglou y Scariot, 2002), mantiene correspondencia con la satisfacción con la vida, señal del grado en el que una persona se siente activa, alerta, con energía y entusiasmo, redunda en este constructo (Sandín, 1999). El humor agresivo, que está relacionado directamente con hostilidad y agresividad (Kuiper y McHale, 2009), mostró correlaciones negativas con la satisfacción con la vida, indicando que un bajo uso del humor agresivo tiene incidencia positiva en la satisfacción con la vida. Además, el humor de descalificación personal, o contraproducente, que busca el uso de un humor cínico a expensas de la propia persona no mostró correlaciones con la satisfacción con la vida. 
Por otra parte, en el análisis de clúster, analizando el uso del humor y la satisfacción con la vida, se obtuvieron tres grupos: uno con un mayor uso del humor en todos sus apartados y una satisfacción con la vida inferior a la media de la muestra; otro, que suponía la mitad de la muestra, que mostraba niveles más bajos en el uso del humor, tanto en los estilos positivos como negativos y con un nivel más bajo en el apartado de satisfacción con la vida; y un tercero en que se encontraron niveles más altos de uso de los humores positivos (afiliativo y de automejora), menor uso del humor contraproducente y un índice mayor de satisfacción con la vida. Estos resultados, indican como el uso del humor está relacionado de alguna forma con la satisfacción con la vida, cuestión apuntada por algunos estudios (Abel, 2002; Dionigi y Gremigni, 2013; Yip y Martin, 2006). Así, un buen uso del humor puede proporcionar soporte emocional, servir en la gestión de conflictos y reportar satisfacción con la vida a los sujetos.

Finalmente, se observó la influencia del uso del humor en la satisfacción con la vida, encontrándose que ser mujer tiene un efecto directo en ésta. Además, el humor afiliativo y de automejora, mostraron un efecto significativo en la satisfacción con la vida de las personas, lo que indicaría el peso en la satisfacción con la vida de los estilos de humor positivos, en línea con otras investigaciones (Maiolino y Kuiper, 2014; Heintz, 2017).

Estos resultados deben ser interpretados teniendo en cuenta las limitaciones de este estudio, que, si bien la muestra es relevante a nivel estadístico, debería ampliarse a otros estratos de la población, dónde podría darse una mayor asociación entre estos constructos: uso del humor y satisfacción con la vida. También serían deseables estudios longitudinales que permitiesen valorar la evolución del uso del humor, así como su vinculación con la satisfacción con la vida, a lo largo de un período más largo de tiempo. Como perspectivas de futuro, conviene señalar la necesidad de implementar programas educativos específicos que trabajen estos constructos en la escuela, por su implicación con la autoestima y el desarrollo de los adolescentes. Los resultados de la investigación nos animan a seguir buscando nuevas preguntas que nos ayuden a definir metodologías y encontrar algunas respuestas, que nos permitan avanzar en la construcción del desarrollo socioafectivo de los adolescentes.

Como conclusión, reflejar que aun cuando los hombres hacen un mayor uso de los estilos del humor, ser mujer y utilizar los estilos de humor afiliativo y de automejora se asocia a una mayor satisfacción con la vida. Además, los datos de la investigación proporcionan evidencia de la relación entre los constructos de estilos del humor y la satisfacción con la vida, pudiendo concluir que el uso cotidiano de los estilos de humor pueden servir para entender mejor nuestra percepción de la satisfacción con la vida. 


\section{REFERENCIAS}

Abel, M.H. (2002). Humor, stress and coping strategies. Humor, 15(4), 365-381.

Aillaud, M., y Piolat, A. (2013). Compréhension et appreciation de l'humour: aproche cognitivo-émotionnelle. Psychologie Française, 58, 255-275. doi:10.1016/j.psfr.2013.04.001

Atienza, F.L., Pons, D., Balaguer, I., y García-Merita, M. (2000). Propiedades psicométricas de la escala de satisfacción con la vida en adolescentes. Psicothema, 12(2), 314-319.

Batool, M., Niazi, S., y Ghayas, S. (2014). Emotional intelligence as a predictor of sense of humor and hope among adults. Journal of the Indian Academy of Applied Psychology, 40(2), 270-278.

Berrios-Martos, M.P., Pulido-Martos, M., Augusto-Landa, J.M., y López-Zafra, E. (2012). La inteligencia emocional y el sentido del humor como variables predictoras del bienestar subjetivo. Behavioral Psychology, 20(1), 211-217.

Cann, A., y Collett, C. (2014). Sense of humor, stable affect, and psychological well-being. Europe's Journal of Psychology, 10(3), 464-479. doi: 10.5964/ejop.v10i3.746

Capps, D. (2006). The psychological benefits of humor. Pastoral Psychology, 54(5), 393-411.

Cassaretto, M., y Martínez, P. (2009). Validación de la Escala del Sentido del Humor en estudiantes universitarios. Revista de Psicología, 27(2), 287-309.

Diener, E., y Seligman, M. (2002). Very happy people. Psychological Science, 13, 81-84.

Diener, E., Suh, E., Lucas, R., y Smith, H. (1999). Subjetive well-being: three Decades of Progress. Psychological Bulletin, 125, 276-302.

Dyck, K., y Holtzman, S. (2013). Understanding humor styles and well-being: the importance of social relationships and gender. Personality and Individual Differences, 55, 53-58. doi:10.1016/j.paid.2013.01.023

Erickson, S., y Feldstein, S. (2007). Adolescent humor and its relationship to coping, defense strategies, psychological distress, and well-being. Child Psychiatry and Human Development, 37, 255-271.

Hay, J. (2000). Emotions of humor in the conversations of men and women. Journal of Pragmatics, 32, 709-742. doi: 10.1016/j.paid.2014.02.024

Heintz, S. (2017). Putting a spotlight on daily humor behaviors: Dimensionality and relationships with personality, subjective well-being, and humor styles. Personality and Individual Differences, 104 (in press).

Kotthoff, H. (2006). Gender and humor: the state of the art. Journal of Pragmatics, 38(1), 4-25. doi: 10.1016/j.pragma.2005.06.003

Kozbetz, A., y Nishioka, K. (2010). Humor comprehension, humor production, and insight: An exploratory study. Humor: International Journal of Humor Research, 23, 375-401. doi:10.1515/humr.2010.017

Kuiper, N.A. (2014). Investigating the role of humor in psychological health and well-being. Europe's Journal of Psychology, 10(3), 464-479. doi: 10.5964/ejop.v10i3.809

Kuiper, N.A., y McHale, N. (2009). Humor styles as mediators between self-evaluative standards and psychological well-being. Journal of Psychology, 143(4), 359-376. doi:10.3200/JRLP.143.4.359-376

Kuiper, N.A., Grimshaw, M., Leite, C., y Kirsh, G. (2004). Humor is not always the best medicine: specific components of sense of humor and psychological well-being. Humor: International Journal of Humor Research, 17, 135-168. doi: 10.1515/humr.2004.002

Maiolino, N.B., y Kuiper, N.A. (2014). Integrating humor and positive psychology approaches to psychological well-being. Europe's Journal of Psychology, 10(3), 557-570. doi:10.5964/ejop.v10i3.753 
Martin, R.A. (2001). Humor, laughter, and physical health: methodological issues and research findings. Psychological Bulletin, 127(4), 504-519.

Martin, R.A., Puhlik-Doris, P., Larsen, G., Gray, J., y Weir, K. (2003). Individual differences in uses of humor and their relation to psychological well-being: Development of the humor styles questionnaire. Journal of Research in Personality, 37, 48-75.

Nezlek, J.B., y Derks, P. (2001). Use of humor as a coping mechanism, psychological adjustment, and social interaction. Humor, 14, 395-413.

Panish, J. (2002). Life satisfaction in the elderly: the role of sexuality, sense of humor, and health. Dissertation Abstracts International, 63(5-B).

Sandin, B., Chorot, P., Lostao, L., Joiner, T.E., Santed, M.A., y Valiente, R.M. (1999). Escalas PANAS de afecto positivo y negativo: validación factorial y convergencia transcultural. Psicothema, 11, 37-51.

Seligman, M., y Csikszentmihalyi, M. (2000). Positive Psychology: An introduction. American Psychologist, 55, 5-14. doi: 10.1037//0003-066X.55.1.5

Seligman, M. (2003). La auténtica felicidad. España: Vergara.

Smedema, S.M., Catalano, D., y Ebener, D.J. (2010). The relationship of coping, self-worth, and subjective well-being: a structural equation model. Rehabilitation Counselling Bulletin, 53(3), 131-142.

Walther, M., y Hilbert, A. (2015). Emotional openness in overweight and normal-weight adolescents. Swiss Journal of Psychology, 71(1), 29-36. doi:10.1024/14210185/a000145

Yip, J.A., y Martin, R.A. (2006). Sense of humor, emotional intelligence, and social competence. Journal of Research in Personality, 40, 1202-1220. doi: 10.1016/j.jrp.2005.08.005

Recibido: 07 de febrero de 2017

Recepción Modificaciones: 19 de mayo de 2017

Aceptado: 21 de mayo de 2017 